\section{Cahiers de Narratologie}

Analyse et théorie narratives

8 | 1997

Création de l'espace et narration littéraire

\title{
Recherches et enquêtes, de Stendhal à Lewis Carroll
}

A Propos de l'espace dans Beatus Ille d'Antonio Munoz Molina

\section{Gérard Lavergne}

\section{OpenEdition}

Journals

Édition électronique

URL : http://journals.openedition.org/narratologie/11597

DOI : 10.4000/narratologie. 11597

ISSN : 1765-307X

Éditeur

LIRCES

Édition imprimée

Date de publication : 1 décembre 1997

Pagination : 223-248

ISBN : 291089746X

ISSN : 0993-8516

\section{Référence électronique}

Gérard Lavergne, "Recherches et enquêtes, de Stendhal à Lewis Carroll », Cahiers de Narratologie [En ligne], 8 | 1997, mis en ligne le 15 décembre 2020, consulté le 25 février 2021. URL : http:// journals.openedition.org/narratologie/11597; DOI : https://doi.org/10.4000/narratologie.11597

Ce document a été généré automatiquement le 25 février 2021.

Article L.111-1 du Code de la propriété intellectuelle. 


\title{
Recherches et enquêtes, de Stendhal à Lewis Carroll
}

\author{
A Propos de l'espace dans Beatus Ille d'Antonio Munoz Molina
}

Gérard Lavergne

1 Nous rappelant la réflexion de Gérard Genette : «Le lieu narratif est fort rarement spécifié, et n'est pour ainsi dire jamais pertinent $»^{2}$ nous voudrions montrer ici comment se construit l'espace dans Beatus Ille ${ }^{3}$ et son importance comme élément générateur de fiction et source de réflexion téléologique.

2 A la gare de Magina l'étudiant Minaya attend le train pour rentrer à Madrid; entre temps il se souvient de son séjour dans la ville. Dans une cellule de la Direction Générale de la Sûreté, après les manifestations des étudiants en 1969, Minaya décide de fuir Madrid et d'aller se réfugier dans sa ville natale Magina. Il rencontre un de ses amis qui lui parle de Jacinto Solana, un poète de la génération de $27^{4}$, tué par la Garde Civile en 1947, et dont il voudrait faire le sujet de sa thèse de doctorat. Minaya demande asile à son oncle Manuel qui vit dans sa maison familiale (p. 25), sous prétexte de vouloir faire, comme thèse de doctorat, la biographie de Solana, vieil ami de son oncle.

3 Minaya, qui était venu peu intéresser par cette thèse, découvre dans la maison, avec les éléments de la biographie de Jacinto Solana, un crime qui s'était produit en mai 1937. Le soi-disant chercheur se fait enquêteur, tout au long d'un véritable voyage initiatique, sur une époque qu'il ne connaissait jusque là que par ouï-dire. Avec lui, le lecteur entreprend son propre voyage initiatique dans l'espace de mémoire que le texte construit.

4 Nous aurons donc une analepse générale, au cours de laquelle le lecteur parcourt, dans un temps et un espace dynamité, l'espace de l'écriture d'une aventure, et l'aventure de cette écriture ${ }^{5}$.

5 Dans une œuvre littéraire, entre les divers éléments qui permettent au lecteur de construire l'espace diégétique, il existe le temps. Beatus Ille est publié en 1986, année du 
cinquantième anniversaire du début de la Guerre Civile, époque que de nombreuses dates reconstruisent dans l'œuvre :

- 1933 (p. 159), Solana rencontre Mariana dont il tombe amoureux.

- 5 juillet 1936 (p. 41), le sculpteur Utrera vient à Magina ; en 1939 il est toujours dans la ville.

-1936-1939, ce sont les années de Guerre Civile.

- 1947 (p. 15), Solana sort de prison. C'est l'année du Référendum sur la Loi de Succession.

-1969, Minaya fuit Madrid. C'est l'année de l'État d'Exception, à cause de la révolte des étudiants.

6 Avec ces dates, nous avons le temps de la fiction qui couvre l'époque d'avant, pendant, et après la Guerre Civile. Minaya, en faisant la biographie de Jacinto Solana, évoque l'espace connoté par cette époque.

$7 \quad$ Le code de lecture peut se définir comme historique.

8 Il est intéressant de remarquer comment le récit commence et finit dans un lieu non spécifié, un non-lieu ${ }^{6}:$ la gare de Magina. On a ainsi l'impression d'un cercle parfait, qui annonce le mouvement centripète de la narration qui va focaliser l'intérêt du lecteur sur un espace spécifique.

9 La Guerre Civile eut comme conséquence de diviser le monde espagnol en deux grands espaces $^{7}$ : l'un (chéma 1) extérieur à l'Espagne, l'autre (schéma 2) intérieur, où l'on distingue de nombreux cercles.

10 Le premier évoque, avec une série de toponymes, des lieux perçus comme centres de rêve, ou au contraire de triste diaspora. Ici (p. 33), Minaya découvre des cartes postales de Solana, du Paris de 1930 et du Moscou de 1935. Paris, c'est le lieu rêvé où allaient se rendre Manuel et sa fiancée Mariana. Moscou, c'est le centre de la Révolution mondiale qui promet des lendemains qui chantent. Là (p. 40) Utrera évoque Rome; la Rome de Michel Ange et de Bernini. Ces toponymes dénotent et connotent un paysage optimiste, intellectuel ou politique, connus du lecteur.

11 Mais, quand Beatriz dit (p. 119) à Solana, son mari, à sa sortie de prison : «j'ai écrit aux amis exilés /.../ tu n'étais pas en France, ni au Mexique, ni en Argentine » (schéma 1), et quand on évoque le camp de concentration d'Argelés, ce n'est pas un monde extérieur d'espérance qui se crée, mais celui de la désespérance.

12 Avec les toponymes « Gibraltar » et «Afrique » (p. 226), apparaissent d'autres espaces ardemment désirés. Ce sont des lieux où pensent arriver Beatriz et ses compagnons. Ils n'y arriveront pas. Tous ces toponymes n'apportent aucune précision historique et n'offrent aucun intérêt diégétique.

Schéma 1 


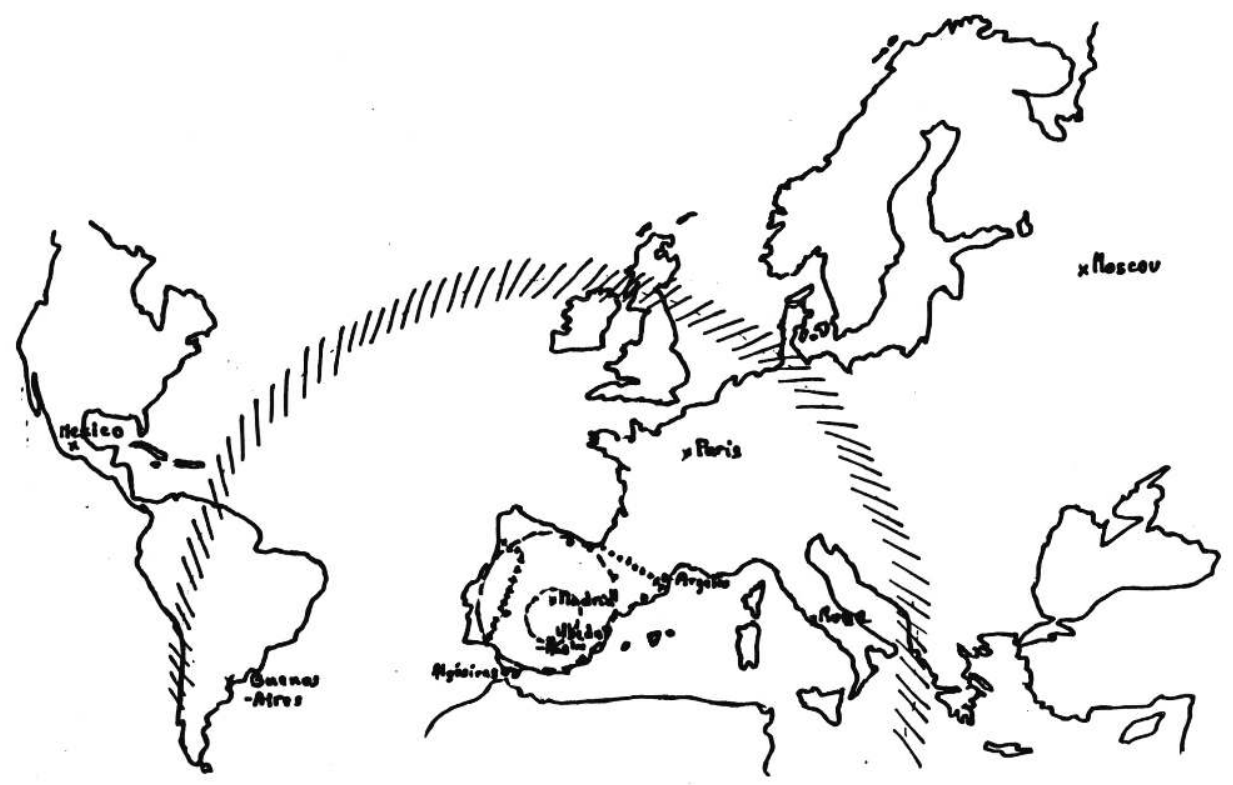

SCHÉMA 2

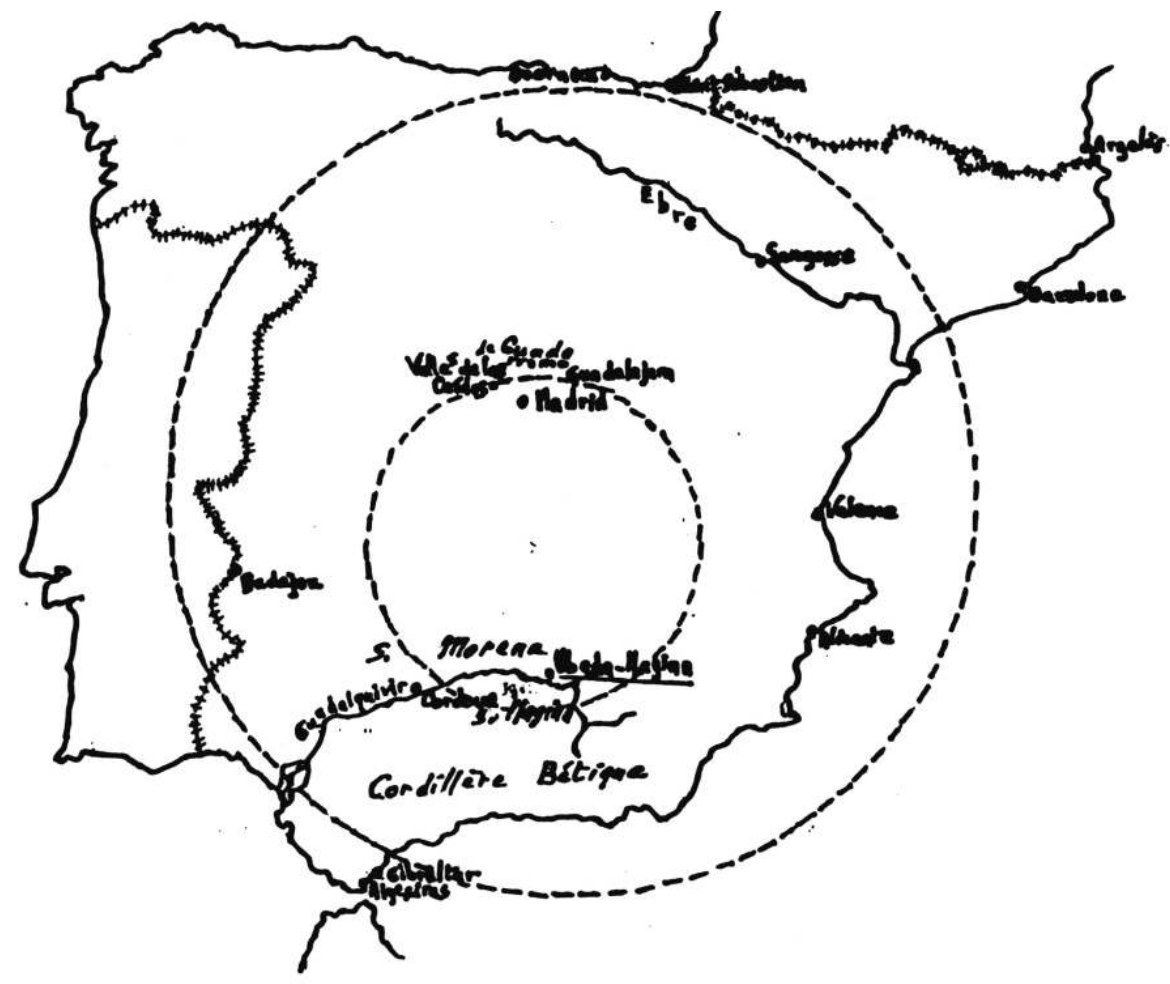

A l'intérieur du pays (schéma 2), de nombreux cercles concentriques se dessinent, matérialisés par des toponymes dont les référents connotent une Histoire mémorisée et mythifiée ; Guernica (p. 177), Saint Sébastien (p. 240), Barcelone (p. 191), Valence et Alicante (p. 32), Badajoz (p. 177) et, évoquée par Beatriz, Algésiras (p. 226) forment le cercle périphérique.

Un autre cercle, intérieur, couvre le centre de la Péninsule : il est déterminé par El Valle de los Caídos (p. 221), la Guadarrama (p. 56), Madrid (p. 12), Guadalajara (p. 30), 
Cordoue (p. 161) et deux fleuves : l'Ebre (p. 32) et le Guadalquivir où furent blessés respectivement Solana et Frasco, contremaître de Manuel, (p. 101).

La narraticité étant pour nous l'aspect phénoménologique d'un élément considéré, c'est-à-dire ce qui, en soi, représente ou métaphorise une réalité connue du lecteur averti, arrivés ici, nous dirons que la narraticité de ces deux isotopies spatiales offre de nombreuses interprétations possibles.

Le lecteur non initié y verra l'évocation du massacre des républicains dans les arènes de Badajoz, le 14 août $1936^{8}$, le bombardement scientifique et cynique de Guernica le 26 avril $1937^{9}$, les luttes acharnées entre les anarchistes et les communistes à Barcelone en mai 1937 (p. 191) ${ }^{10}$, les assassinats et les lynchages des deux côtés (p. 166), le Congrès des écrivains antifascistes pour la défense de la culture et de la littérature de qualité à Valence.

Mais le lecteur attentif ${ }^{11}$ remarquera que si ces toponymes, éléments d'une mimésis géographique, font surgir d'une mémoire collective des souvenirs historiques, créant une forte impression de réalité, l'intérêt premier de ces macro-espaces est de tisser une toile de fond faite d'historicité, où sera proposée une réflexion métaphysique sur les réalités du monde. A ce moment, ce même lecteur aura parcouru une partie de son propre voyage initiatique, et sera arrivé au cœur du texte, à l'imaginaire Magina, espace d'une saga éternelle.

Vue depuis la vallée du Guadalquivir, Magina prend la consistance ésotérique d'un monde fait de rêve et de réalité. Son espace entre brusquement dans l'imaginaire du lecteur, surgissant des lignes du texte, emporté par la voix discrète et poétique des didascalies et la symbolique des couleurs.

-p. 55 : Mágina vue du Sud.

-p. 48: «Mágina, dressée sur la proue immobile d'une colline trop éloignée du Guadalquivir.» (photo 1)

-p. 17 : «Maginaville intacte, offerte et distante sur sa colline bleue $»^{12}$

18 Avec ces didascalies, Magina, sur son promontoire, dans un ciel d'azur, couleur de rêve $^{13}$, ne manquera pas d'évoquer un autre centre de voix éternelle qui a pu servir de référent : le théâtre d'Epidaure sur son promontoire.

19 Minaya, en arrivant et en sortant de la gare, entre en un lieu qu'il va parcourir au cours de son enquête. Avec lui, le lecteur, suivant le fil du texte, découvre peu à peu les espaces qui surgissent des pérégrinations de l'étudiant, et le sens de ces espaces.

C'est ainsi qu'apparaissent ces toponymes qui n'occultent pas le référent: la place de l'église San Pedro (p. 9), la maison aux deux heurtoirs de bronze (p. 9) ${ }^{14}$ (photo 2), la place du général Orduña et sa statue (p. 15, 56), le monument aux morts (Caídos) (p. 68) (photo 3), la Tour de l'Horloge (p. 53) (photo 4), la montagne Magina d'où la ville fictionnelle tire son nom, au sud du Guadalquivir (p. 56), la rue Lune et Soleil (p. 61), les ruelles, souvent pavées, de Magina (p. 172)... le lecteur qui connait l'Andalousie aura reconnu avec cette topographie mimétique, quasi homologue, la ville de la Renaissance Ubeda (Jaen) ${ }^{15}$

21 Le rôle d'une telle mimésis ? Henri Mitterand l'indique dans Le Discours du roman quand il dit: « le nom des lieux proclame l'authenticité de l'aventure par une sorte de reflet métonymique qui court-circuite la suspicion du lecteur : puisque le lieu est vrai, tout ce qui lui est contigu, associé, est vrai » ${ }^{16}$. 
22 L'homologie donne donc une forte illusion de réalité ; c'est l'effet de réel de Barthes ${ }^{17}$. Cependant, c'est une œuvre de fiction, et l'auteur reconstruit son propre espace. Il parle de train, de gare, il n'y a jamais eu de rails ni de train (p. 8) ${ }^{18}$ (photo 5).

23 La place du général Orduña n'a pas existé, mais la place d'Andalousie oui. On y trouve la statue «fusillée» pendant la guerre par les Gardes d'Assaut (p. 56). Ce n'est pas la 
statue du général Orduña, personnage de fiction, mais celle du Général Saro, héros de la guerre du $\operatorname{Maroc}^{19}$ (photo 6).

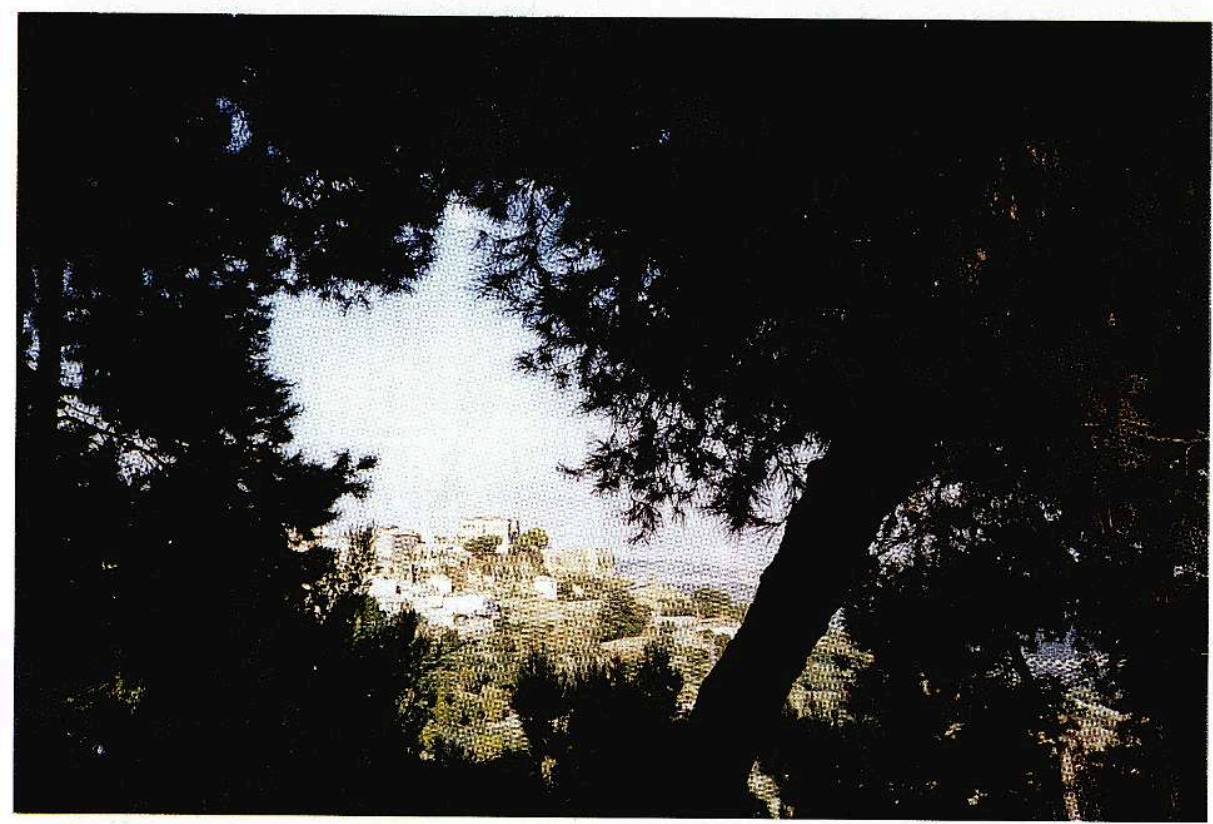

№ 1 Magina - Ubeda (Jaén)

dressée sur la proue immobile... (p. 48)... distante sur sa colline bleue (p. 17). Référent

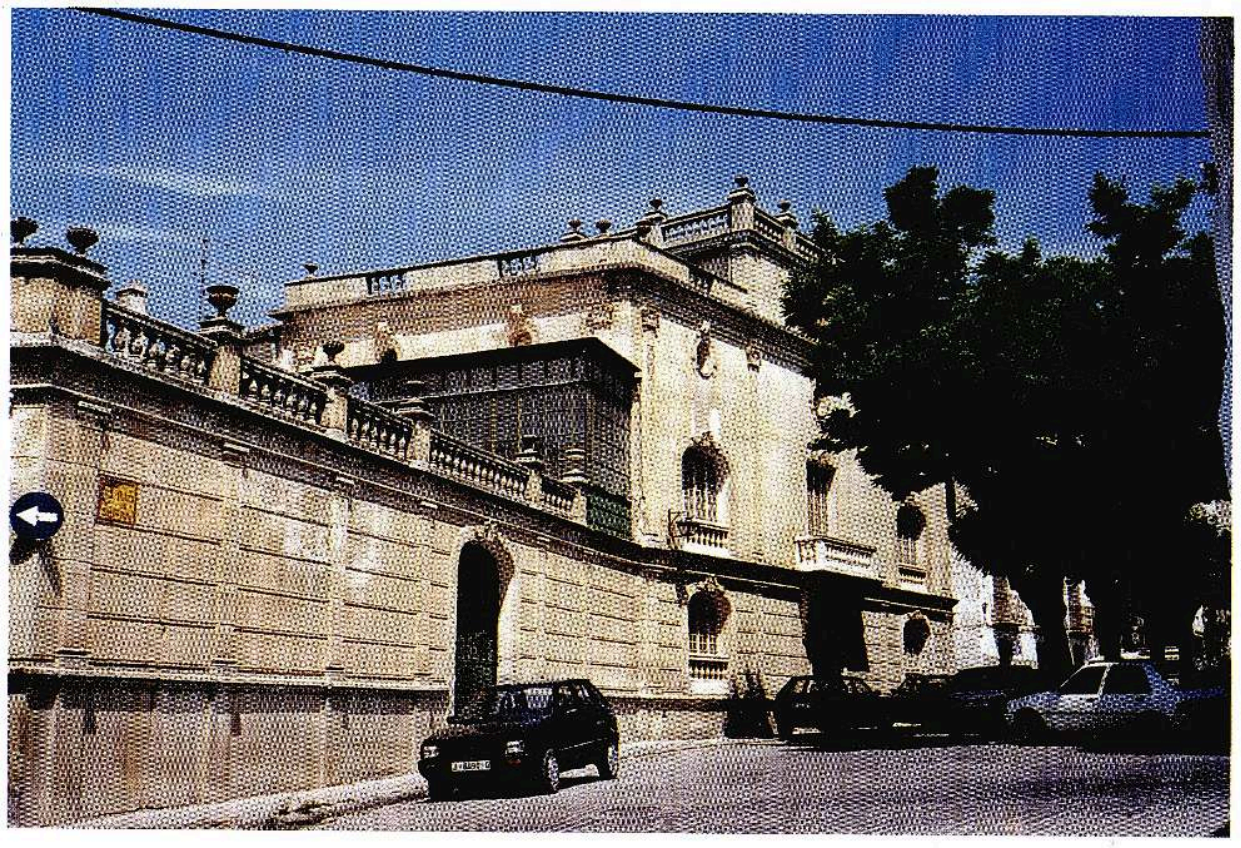

№ 2 La maison aux heurtois de bronze (p. 9). Référent 


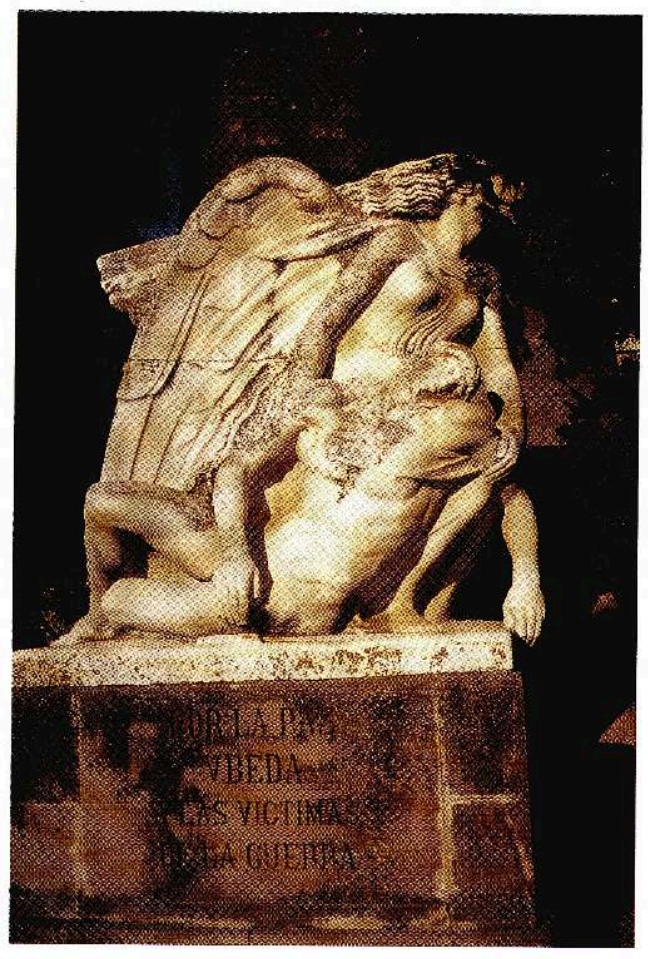

№ 3 Le monument aux morts (à « los Caídos »)

le visage tourné vers le mur (p. $40 ; 64)$.

Référent et déviance narrative

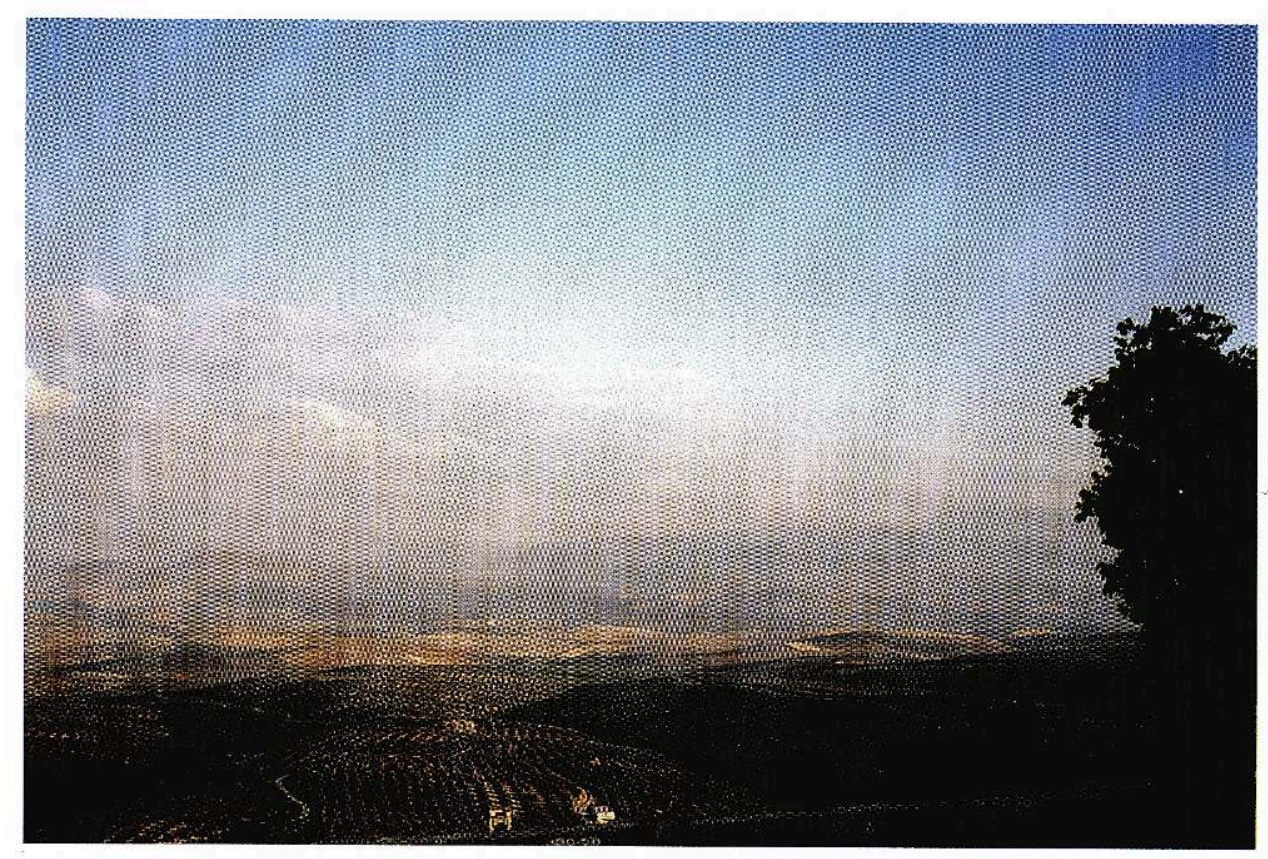

№ 4 La sierra Magina (p. 56). Référent 


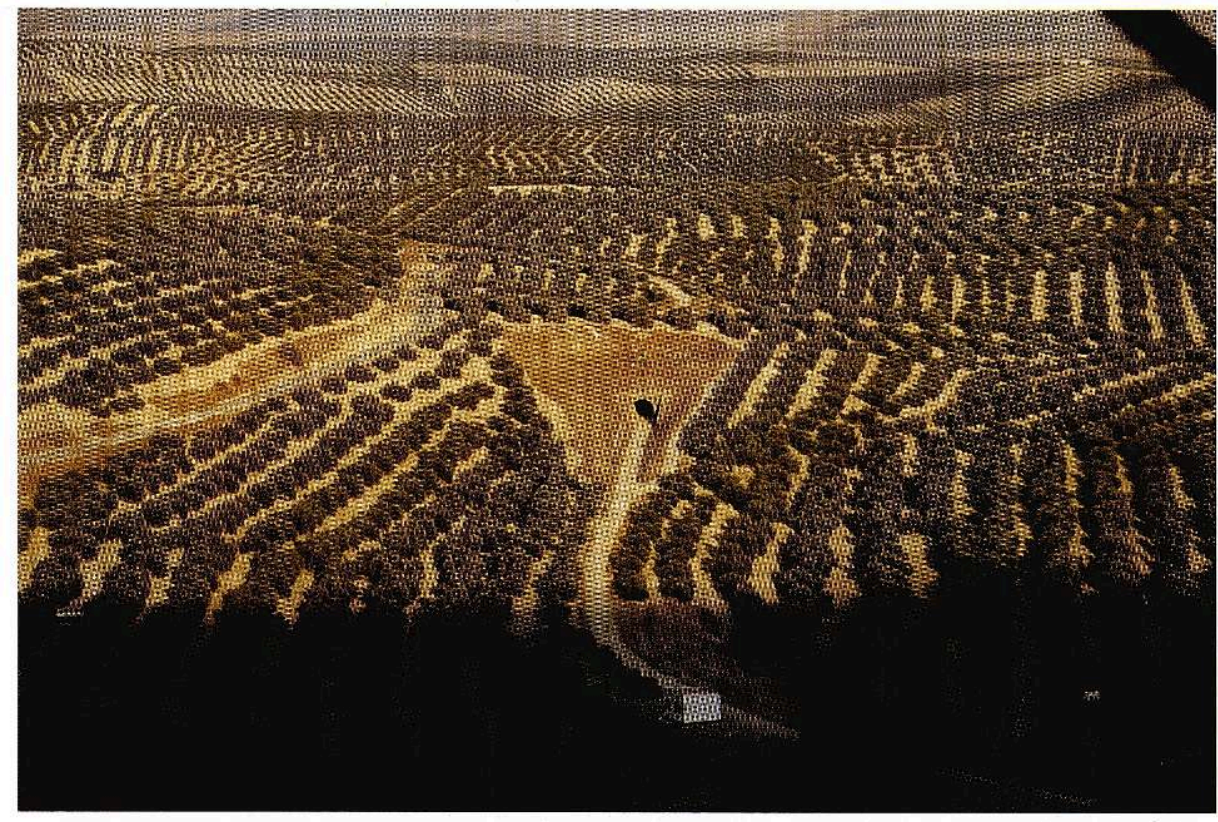

$N^{\circ} 5$ Minaya attend le train (p.8). Référent et déviance topographique.

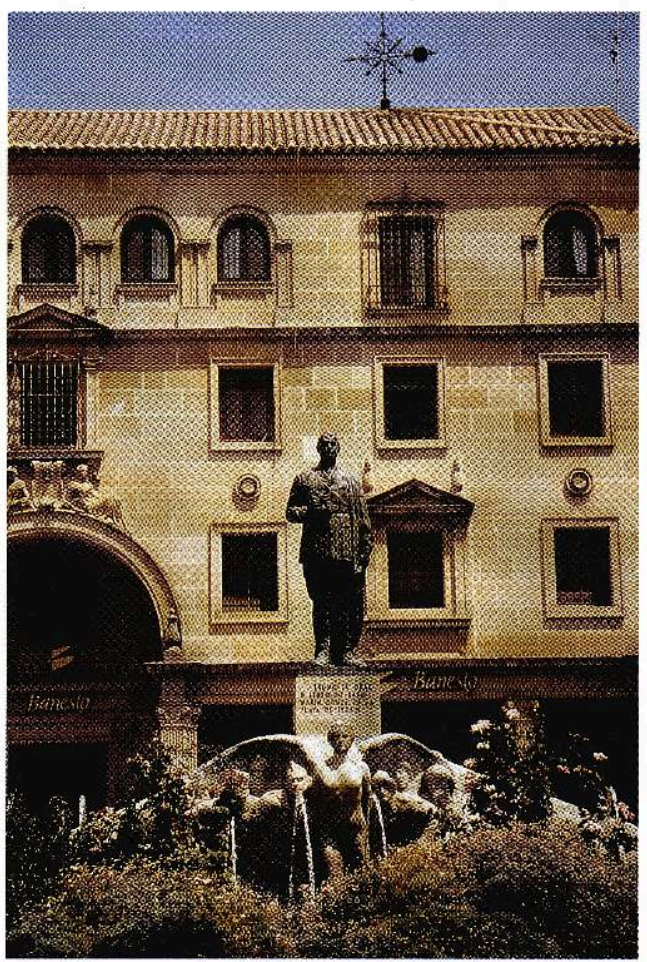

Nº 6 La statue fusillée du général Saro (p. 15-56). Référent

24 Sur la place des morts à la guerre (Caídos), aujourd'hui de la Mairie, le monument aux morts existe, dans le même espace, mais avec une dédicace moins politique : « Pour la paix, Ubeda aux victimes de la guerre $»^{20}$.

25 Le mouvement du corps du héros dans la fiction ne correspond pas à celui du monument. 
Nous voyons ainsi apparaître des traits de déviance de la topographie mimétique qui mettront en évidence la valeur actancielle de l'espace dénoté.

Le lecteur remarque vite que la fiction à Magina tourne autour de trois espaces: la place San Pedro avec la maison de Manuel, la place des morts où vivent Inés et son parrain Solana, l'«Ile de Cuba », propriété de Manuel sur les rives du Guadalquivir, où vint travailler et mourir Solana en 1947, lieu qui finit par être réel de par le vérisme de la topographie mimétique environnante.

Nous pouvons rappeler maintenant Philippe Hamon qui, dans un article sur « le savoir dans le texte », parle d'une classe de lieux qu'il appelle « cybernétiques » c'est-à-dire «les endroits où »se trouve, s'échange, se met en forme l'information »; et il donne comme exemple: « les salons de rencontre, les lieux d'où l'on observe un spectacle $»^{21}$. Ces espaces, en général clos, souvent objet d'infraction d'un code éthico-social, et par suite moteur de la fiction, dominent dans l'œuvre.

Dans la propriété de Manuel, Minaya et Inés vont à la recherche de documents. Ils découvrent, dans l'espace fermé d'un pailler, une malle, et à l'intérieur un cahier (p. 102). C'est le cahier journal apocryphe du mort. De ce texte va naitre la majeure partie d'une biographie, fausse puisque la découverte et la narraticité de la pièce font croire à Minaya qu'il a découvert un document historique authentique ${ }^{22}$. Sur la place où se trouve la statue dédiée aux morts, Inés, conduisant Minaya derrière le piédestal, l'invite à s'accroupir pour observer le visage du mort. Il semble dormir «le visage vers le mur ». C'est une femme. Au front elle porte un cercle : la marque d'un assassinat (p. 64). De cette place, centrée sur une statue au mouvement dissimulateur, surgit la problématique : Qui est-ce ? Le biographe se fait détective. Il change de statut. La piste de lecture change également : nous sommes dans l'intrigue policière.

Sur la même place, Minaya entre dans la maison où vivent Inés et son parrain, et il voit Solana qui n'est pas mort. Blessé en 1947, celui-ci fut recueilli dans une ferme où il s'occupa d'Inés. Pour éviter la répression, il entra dans l'anonymat. Son refuge est l'origine des manipulations et des déplacements que connut Minaya, et avec lui le lecteur, imaginés par Solana et réalisés avec la complicité d'Inés. De cet espace naît, écrit par Minaya, le Beatus Ille $2^{\mathrm{e}}$, roman spéculane du Beatus Ille $1^{\mathrm{e}}$. Ainsi naît la biographie apocryphe d'un poète-soldat de la 2de République.

Étudions maintenant ce que nous appellerons la narrativité, ou plutôt l'actorisation du lieu où vit Solana ${ }^{23}$. Cet espace est celui qui explique et rend vraisemblable toute la diégèse, ce que confirme Solana quand il dit «construisons-lui le labyrinthe qu'il désire... les pas qui l'amèneront à trouver le roman et à découvrir le crime. Il suffisait d'envoyer Inés chez un imprimeur » (p. 276) ${ }^{24}$.

C'est ainsi que cet espace conditionne par lui-même l'existence du personnage d'Inés, la structure et la progression de la fiction, et génère la présence d'un narrateur absent ambigu. L'espace n'est plus un lieu avec lequel on agit, mais un lieu qui agit.

Entrons dans la maison de Manuel Crivelli, dont nous évoquerons en premier lieu la narraticité des espaces, tous clos.

La pièce où vit Doña Elvira est au dernier étage : c'est un lieu clos. Là, Minaya la rencontre au milieu de "vêtements et de meubles d'un autre âge» (p. 70) ${ }^{25}$. Elle a toujours sa télévision allumée, mais elle ne la regarde ni ne l'écoute (p. 35). Le monde extérieur lui est indifférent. Défenseur de la tradition, elle est l'allégorie d'une société enfermée dans ses coutumes. Mais si c'est ce que nous pouvons déduire de la narraticité 
de cet endroit, on peut déjà prévoir que ce lieu, en plus de la métaphorisation d'une personnalité, sert de tour de guet pour observer ce qui se passe dans le jardin et déterminer ainsi diverses fonctions diégétiques.

Dans la bibliothèque de la maison il y a beaucoup de livres. Ceux-ci éveillent la convoitise de Solana et son désir d'écrire "pour être riche /.../ implacable» (p. 50 ${ }^{26}$. Là, Minaya rencontre la photographie d'une femme entre ses deux hommes. L'ekphrasis annonce l'avenir ; là, Orlando, comme Satan sur la montagne, tente de nuit Solana: il lui dit que Mariana l'attend dans le jardin (p. 178). La noce est pour le lendemain. La bibliothèque donc, lieu des plus cybernétiques, participe aux péripéties de la fiction et à sa dramatisation.

L'espace du jardin de la maison favorise la liaison amoureuse entre Jacinto et Mariana. Mais là-haut, dans un espace clos, «la lumière jaillit ». Quelqu'un les épiait (p. 86). C'est, depuis sa chambre-tour de guet, Doña Elvira. La narrativité de l'espace est donc l'élément adjuvant qui prépare le continuum fictionnel.

37 La chambre nuptiale de Manuel et Mariana, prête depuis 1937, comme pour servir à nouveau, est en soi un espace clos. Manuel en interdit l'entrée à tous. Si sa narraticité éclaire sur l'idiosyncrasie du personnage mort-vivant depuis la mort de sa fiancée en 1937, sa narrativité fait que Manuel y surprend, en 1969, les amours de Minaya et d'Inés. «Alors /.../ le temps se déchira comme si une pierre vengeresse avait brisé les miroirs qui les reflétaient» (p. 111) ${ }^{27}$. Manuel tombe foudroyé, en notant qu'il avait préféré le souvenir à la réalité de la vie. Il a fallu pour cela que soit violé l'espace miroir de son souvenir.

Nous avons vu que dans Beatus Ille l'espace est un véritable acteur, générateur de fiction $^{28}$, qui marque par sa structuration mimétique, quasi homologue, tout ce qu'il touche d'une forte impression de réalité. Le roman peut donc paraitre comme «le miroir que l'on promène le long du chemin ".

Mais laissons là Stendhal. Évitant le mirage de la réalité historique que nous présente l'œuvre, suivons avec Lewis Carroll Alice « dans le couloir imaginaire qui se prolongeait de l'autre côté du miroir » (p. 14) ${ }^{29}$.

Derrière le miroir que voyons-nous surgir ? En premier lieu apparaît la finalité de la fiction littéraire : susciter l'intérêt. Utrera révèle à Minaya le mystère de la statue, lui demandant le secret. Minaya promet : mais déjà « imaginant /.../ comment il racontera ces choses à Inés /.../ pour faire naître dans ses yeux l'éclat d'un mystère séduisant » (p. 95) ${ }^{30}$. Ailleurs on voit comment, par manque de documents, le chercheur veut inventer, « ourdir le personnage ambigu d'un héros » (p. 231$)^{31}$.

41 A l'heure de l'anagnorésis, le narrateur narrant rencontre le narrateur narré, dans l'espace clos d'où a surgi la mystification, qui se résume ainsi : «donnons-lui non la vérité, mais ce que lui a supposé s'être produit » (p. 276) ${ }^{30}$.

De cette façon, derrière le miroir, le lecteur découvre en réalité un ars escribendi, dans une isotopie faite d'éléments quasi subliminaux, l'art de rédiger un ouvrage qui suscite l'intérêt, même si c'est une thèse historique en définitive trompeuse.

Il apparait ainsi une réflexion téléologique qui prend forme avec les personnages.

- Mariana est la femme républicaine ; Elvira ne l'apprécie guère. Aimée de Manuel, elle occupe un espace interdit car il a déjà une fiancée. Elle meurt assassinée par Utrera : 
Elvira le lui a ordonné. Il semble là que deux mondes s'opposent : Mariana, allégorie de la Nouvelle Espagne, et Elvira, allégorie de la réaction.

Mais, passons derrière le miroir, la réalité est autre: Mariana, allégorie de la République, meurt parce que ceux qui l'aimaient n'ont pas su la défendre, et Elvira exécute Mariana car celle-ci, si elle partait à Paris avec Manuel, elle l'éloignerait de sa mère. Elvira se révèle ainsi une mère castratrice qui se venge.

- Solana lui, sera dans la thèse de Minaya un héraut, un paladin de la République populaire, du peuple. Mais on découvre tout au long du texte son caractère envieux de tout ce qui l'entoure. Sa femme Beatriz, lui déclare qu'« il n'avait rien trahi, parce qu'il n'y avait jamais rien eu à quoi il fut loyal » (p. 276) ${ }^{32}$. Tel est le héros qui va entrer, avec une biographie inventée, au panthéon des gloires hispaniques. Signalons sans insister, que, Mariana et Solana ayant voulu franchir le seuil d'un espace qui leur était interdit, et dont ils ont voulu enfreindre les règles éthico-sociales, cet espace les expulse tous les deux. On peut dire la même chose d'une certaine façon de Minaya en train d'attendre le train, expulsé, d'abord de Madrid, et maintenant de Magina.

Nous pourrions parler d'une stratégie déceptive, dysphorique; il vaudrait mieux voir ici une véritable parabole, lancée depuis le promontoire de la création littéraire. En effet, à la fin de sa recherche, Minaya abandonne l'espace de Magina, l'Espagne par antonomase, pour entrer avec sa thèse dans le champ de la littérature. Mais son œuvre peut être une grande leçon de déontologie car elle rappelle que les conflits ne naissent pas toujours des idéologies, mais souvent à cause des passions qui agitent l'homme.

En passant derrière le miroir de la réalité, en soulevant les couvercles qui protègent les espaces fermés, métaphores du cœur humain, le lecteur a vu surgir l'envie, la jalousie, la haine, l'amour, et il aura compris peut-être que les documents, les textes historiques fondateurs, doivent être lus avec attention et qu'il faut relativiser tout ce que l'on y affirme, pour que l'espace qu'ils évoquent ne devienne pas un monde insupportable.

Après avoir étudié dans Beatus Ille la construction de l'espace, nous avons vu comment celui-ci, dans une narration littéraire, peut être un acteur parfaitement précisé et tout à fait pertinent.

\section{NOTES}

2. GenetTe Gérard, Figures III, Paris, Seuil, 1977, p. 228.

3. Munoz Molina Antonio : Beatus Ille, Madrid, Seix Barral, 1986, 281 p. sera désigné dans la suite des notes par B.I.

4. En évoquant le personnage de Jacinto Solana, nous parlerons de poète de la génération de 27 , d'après les déclarations de l'auteur lui-même, qui annonce en particulier dans le discours prononcé lors de son admission à l'Académie Royale Espagnole (R.A.E.) : «Sans Josep Torres Campalans, sans ce roman de Max Aub, je n'aurais pas écrit l'histoire d'écrivains de la génération de 27 » (El País 15-6-96, Babelia p. 9).

5. Nous parodions Jean Ricardou qui parla de «l'écriture d'une aventure et de l'aventure d'une écriture » dans Pour une théorie du Nouveau Roman, Paris, Seuil, 1971, p. 32. 
6. Cf. AUGE Marc : Non-lieu. Introduction à une anthropologie de la surmodernité. Evreux, Seuil, 1992.

7. Voir les schémas 1 et 2 et les photographies pp. 226-227. éléments qui ont été projetés lors de la communication faite à Séville. Photographies de G. Lavergne.

8. THOMAs Hugh : La Guerre d'Espagne, Paris, R. Laffont, 1964, p. 255.

9. Ibid., p. 425.

10. Ibid., p. 430 et s.

11. Vincent JouvE parlerait de lectant. Cf. son ouvrage : L'Effet personnage dans le Roman, Paris PUF, 1992, p. 83 et ss.

12. B.I. « Mágina, detenida y alta en la proa de una colina demasiado lejos del Guadalquivir » (p.

48) « Mágina... ciudad intacta, ofrecida y distante sobre su colina azul » (p. 17)

13. Le bleu est le chemin du rêve. Cf. Jean Chevalier, Alain GeErbrant : Dictionnaire des symboles, Paris, Laffont, 1994, p. 129.

14. La demeure qui sert de référent est au $\mathrm{n}^{\circ} 2$ de la Place San Pedro.

15. Ubeda (Jaen), ville natale de l'auteur; ses parents, d'anciens maraîchers y vivent encore. Ubeda, comme le théâtre d'Epidaure se dresse devant un panorama montagneux. C'est la plaine du Guadalquivir et au Sud on voit la montagne Magina.

16. MitTERAND Henri : Le Discours du Roman, Paris, PUF écriture, 1980, p. 194.

17. B ARTHES Roland: (L'effet de réel) «El efecto de realidad», Comunicaciones, Madrid, Tiempo Contemporáneo, 1971, p. 95-102.

18. Cf. Guerrero Juan Pasqua : "Les Communications de jadis. Rêves de chemins de fer et réalité de tramway » Biographie de Ubeda p. 496-500.

19. Le général Saro, une personnalité d'Ubeda, fut le héros de la plage

d'Ixdain, quand eut lieu le débarquement des troupes espagnoles au Maroc. Il fit partie du directoire du Dictateur Miguel Primo de Rivera. Il fut exécuté à Madrid en 1936. La statue était autrefois sur la place du $1^{\mathrm{er}}$ mai, où elle fut remplacée par celle de Saint Jean de la Croix. Le général Saro aujourd'hui se trouve avec la tour de l'horloge sur la Place d'Andalousie. Comme il était déjà mort, les gardes d'Assaut fusillèrent sa statue à Ubeda. cf Guía de Ubeda, ciudad del Renacimiento, Buena, La Loma, 1995, p. 53.

20. Comme l'expression « Los Caídos » (Les Morts, les Tombés à la guerre) offrait une connotation franquiste, on lui substitua la dédicace actuelle à tous les Morts du conflit : « Pour la paix, Ubeda aux victimes de la Guerre ».

21. Hamon Philippe : «Le savoir dans le texte », Revue des Sciences Humaines n 4, 1975, p. 489-499, cité par Henri Mitterand, op. cit., p. 193.

22. RicoeUR Paul: Temps et récit, T. III : Le Temps raconté, Paris, Seuil, 1985, p. 215. L'auteur rappelle l'existence d'une critique historique basée sur des documents à finalité déclarée.

23. MitTerAnd Henri, op. cit., p. 211 parle «d'actancialisation de l'espace » et il dit: « Lorsque le circonstant spatial /.../ devient à lui seul d'une part la matière, le support, le déclencheur de l'événement, et d'autre part l'objet idéologique principal, peut-on encore parler de circonstant, ou, en d'autres termes, de décor? Quand l'espace romanesque devient une forme qui gouverne par sa structure propre, et par les relations qu'elle engendre, le fonctionnement diégétique et symbolique du récit, il ne peut rester l'objet d'une théorie de la description » et il ajoute p. 212 : «Le roman, depuis Balzac surtout, narrativise l'espace, au sens précis du terme : il en fait une composante essentielle de la machine narrative ». Plutôt qu'actancialisation, nous préférons utiliser le mot

« actorisation » car les deux termes actant et acteur n'ont pas la même valeur sémantique.

24. B.I. p. 276 : «Construyámosle el laberinto que desea /.../ los pasos que lo lleven a encontrar la novela y descubrir el crimen. Bastaba mandar a Inés a una imprenta. »

25. B.I. p. 70 : « las ropas y muebles de otro tiempo».

26. B.I. p. 50 : « para ser rico /.../ implacable». 
27. B.I. p. 111 : «entonces /.../ se rasgó el tiempo como si una piedra vengativa hubiera roto los espejos que los reflejaban ».

28. Greimas A. -J., Courtes J.: Sémiotique. Dictionnaire raisonné de la théorie du langage, Paris, Hachette, 1993, p. 249 : «Dans le projet sémiotique qui est le nôtre, la narrativité généralisée libérée de son sens restrictif qui la liait aux formes figuratives des récits - est considérée comme le principe organisateur de tout discours. »

29. B.I. p. 14 : « en el corredor imaginario que se prolongaba al otro lado del cristal ».

30. B.I. p. 95 : « contarlas a Inés para incitar en sus ojos un brillo de apetecido misterio ».

31. B.I. p. 231 : « quiso urdir la figura ambigua de un héroe », p. 276 : « démosle no la verdad sino lo que él supone que sucedió »

32. B.I. p. 271 : « no había traicionado nada porque nunca hubo nada a lo que fuera leal... ».

\section{AUTEUR}

\section{GÉRARD LAVERGNE}

Université de Nice-Sophia Antipolis 